\title{
VEGI-armed oncolytic adenovirus inhibits tumor neovascularization and directly induces mitochondria-mediated cancer cell apoptosis
}

\author{
Tian Xiao ${ }^{1, *}$, Jun Kai Fan ${ }^{1, *}$, Hong Ling Huang ${ }^{1}$, Jin Fa Gu${ }^{1}$, Lu-Yuan $\mathrm{Li}^{2}$, Xin Yuan Liu ${ }^{1,3}$ \\ ${ }^{I}$ Laboratory of Molecular Cell Biology, Institute of Biochemistry and Cell Biology, Shanghai Institutes for Biological Sciences, \\ Chinese Academy of Sciences, Shanghai 200031, China; ${ }^{2}$ Department of Pathology, University of Pittsburgh Medical Center, Pitts- \\ burgh, PA 15213, USA, ${ }^{3}$ Xinyuan Institute of Medicine and Biotechnology, Zhejiang Sci-Tech University, Hangzhou 310018, China
}

Vascular endothelial cell growth inhibitor (VEGI) is a member of the tumor necrosis factor superfamily and plays an important role in vascular homeostasis. In this study, to investigate the anticancer therapeutic potential of this gene, a secreted isoform of VEGI (VEGI-251) was inserted into a selectively replicating adenovirus with E1B $55 \mathrm{kDa}$ gene deletion (ZD55) to construct ZD55-VEGI-251. We report here that secreted VEGI-251 produced from ZD55VEGI-251-infected cancer cells potently inhibits endothelial cell proliferation, tube formation in vitro and angiogenesis of chick chorioallantoic membrane in vivo. Additionally, ZD55-VEGI-251 infection leads to a much more severe cytopathic effect than control viruses on several human cancer cell lines, including cervical cancer cell line HeLa, hepatoma cell line SMMC-7721 and colorectal cancer cell line SW620. Further study reveals that the increased cytotoxicity is a result of VEGI-251 autocrine-dependent, mitochondria-mediated apoptosis accompanied by caspase-9 activation, enhanced caspase-3 activation and PARP cleavage. Moreover, ZD55-VEGI-251-treatment of athymic nude mice bearing human cervical and colorectal tumor xenografts markedly suppressed tumor growth. Our findings indicate that the combined effect of antiangiogenesis and apoptosis-induction activity makes the VEGI-251-armed oncolytic adenovirus a promising therapeutic agent for cancer.

Keywords: VEGI-251, oncolytic adenovirus, antiangiogenesis, apoptosis, tumor therapy

Cell Research (2010) 20:367-378. doi: 10.1038/cr.2009.126; published online 17 November 2009

\section{Introduction}

Angiogenesis plays an important role in the progression of tumors from dormancy to a clinically relevant cancer $[1,2]$. Tumor-induced proliferation of new blood vessels has a profound effect on tumor growth and metastasis [3]. Therefore, anti-vascular strategies remain highly attractive approaches for the development of novel anticancer therapeutics $[4,5]$.

\footnotetext{
*These two authors contributed equally to this work. Correspondence: Xin Yuan Liu ${ }^{\mathrm{a}}$, Lu-Yuan $\mathrm{Li}^{\mathrm{b}}$

${ }^{\mathrm{a}}$ Tel: +86 21 54921126; Fax: +86 2154921126

E-mail: xyliu@sibs.ac.cn

${ }^{\mathrm{b}}$ Tel: +412 623 1118; Fax: +412623 4747

E-mail: 1il@upmc.edu

Received 14 April 2009; revised 3 September 2009; accepted 8 September 2009; published online 17 November 2009
}

Vascular endothelial cell growth inhibitor (VEGI) is a multifunctional gene that belongs to the tumor necrosis factor (TNF) superfamily (TNFSF15), and is expressed predominantly in endothelial cells [6]. Recombinant VEGI exhibits two distinctly different activities on endothelial cells: early G1 arrest of G0/G1-cells responding to growth stimuli and programmed death of proliferating cells [7], but it does not affect the growth of nonendothelial cells such as human vascular smooth muscle cells and human breast cancer cell line, MDA-MB-231 [8]. One of three identified VEGI isoforms, VEGI-251 (also known as TL1A), of which the $\mathrm{NH}_{2}$ terminus contains a secretion signal peptide, functions as an autocrine cytokine to stabilize the vasculature [9]. Overexpression of VEGI-251 results in potent inhibition of endothelial cell proliferation, angiogenesis and tumor growth [10]. Reduced VEGI expression is reported to be associated with poor prognosis in breast cancer patients [11]. Other 
activities of VEGI also have been reported, including the stimulation of T-cell activation [12] and dendritic cell maturation [13]. Moreover, TNFSF15 single nucleotide polymorphisms are suggested to contribute to the susceptibility of inflammatory bowel disease [14, 15].

Previous researches demonstrated that an oncolytic adenoviral system, the engineered ZD55-gene system, could be used as a potent antitumor strategy [16-19]. In this study, considering that angiogenesis plays a central role in tumor growth, we investigated the therapeutic potential of a combination of the antiangiogenic ability of VEGI-251 and the oncolytic activity of the ZD55gene system. Thus, ZD55-VEGI-251 was constructed by inserting the VEGI-251 gene expression cassette into the oncolytic adenoviral vector ZD55. Then, its effect on endothelial cells and cancer cells in vitro and antitumor activity in vivo were determined. We report here that, in addition to the anticipated paracrine-dependent antiangiogenesis effect of VEGI, ZD55-VEGI-251 infection gives rise to an autocrine-dependent, mitochondria-mediated apoptosis effect of VEGI-251 on the cancer cells. Our findings suggest that ZD55-VEGI-251 is a highly promising therapeutic agent for cancer.

\section{Results}

\section{Characterization of ZD55-VEGI-25I}

ZD55 is an oncolytic serotype 5 adenovirus with deletion of the E1B $55 \mathrm{kDa}$ gene [19], which is similar to
ONYX-015. Here, with the goal of efficient delivery and expression of VEGI-251, a VEGI-251 expression cassette was inserted into ZD55 to construct ZD55VEGI-251 (Figure 1A). To identify ZD55-VEGI-251 and other recombinant adenoviruses (Ad-VEGI-251, ZD55EGFP, ZD55-TRAIL), human cervical cancer cell line HeLa was infected with these viruses and the expression of E1A and E1B $55 \mathrm{kDa}$ proteins was examined (Figure 1B). Like ONYX-015, ZD55-VEGI-251 and other ZD55-gene viruses express the E1A protein, but failed to express the E1B $55 \mathrm{kDa}$ protein. In contrast, wild-type adenovirus and HEK293 (human embryonic kidney cell line) cells can express both proteins, whereas the replication-deficient Ad-VEGI-251 expresses neither of them. We further determined whether VEGI-251 was expressed and secreted by collecting the conditioned medium (CM) of the infected cells and examining the expression of VEGI-251 by western blot. As shown in Figure 1C, $48 \mathrm{~h}$ after being infected with ZD55-VEGI-251 at a multiplicity of infection (MOI) of 10, VEGI-251 can be detected in the $\mathrm{CM}$ of HeLa cells. These results indicate that the construction of ZD55-VEGI-251 is correct and ensure it for further studies.

Inhibition of proliferation and tube formation of human umbilical vein endothelial cells (HUVECs)

To investigate the anti-proliferation effect of ZD55VEGI-251 on endothelial cells, primary cultured HUVECs were infected with ZD55-VEGI-251 or ZD55-
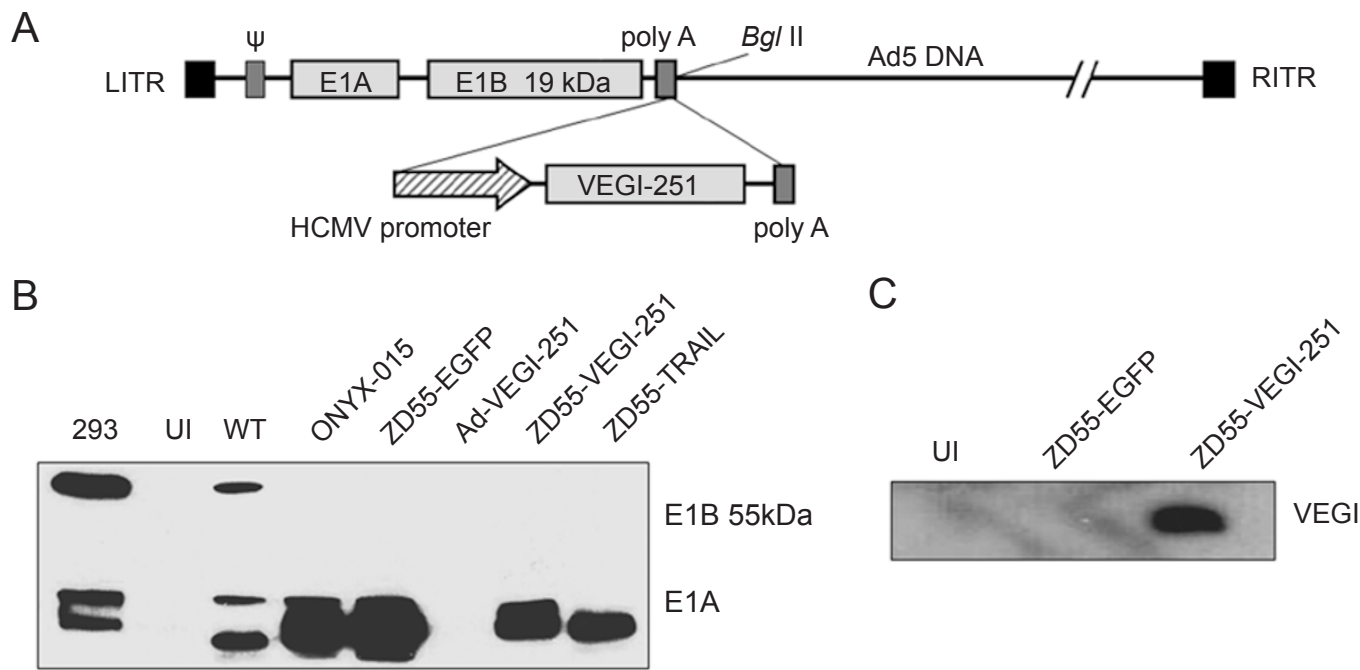

Figure 1 Characterization of ZD55-VEGI-251 and other recombinant adenoviruses. (A) A schematic drawing of the oncolytic adenovirus ZD55-VEGI-251. $\psi$ is the encapsidation signal; LITR (or RITR) are abbreviations for left (or right) inverted terminal repeat. (B) Identification of different adenoviruses by western blot. The cell lysates of HeLa cells infected with different adenoviruses were prepared for analyzing the expression of adenovirus E1A and E1B $55 \mathrm{kDa}$ proteins. 293 cells lysate was used as positive control. UI, uninfected HeLa cells; WT, wild-type adenovirus. (C) Secreted VEGI-251 in the medium of HeLa cells infected with ZD55-EGFP or ZD55-VEGI-251 was detected by western blot. 

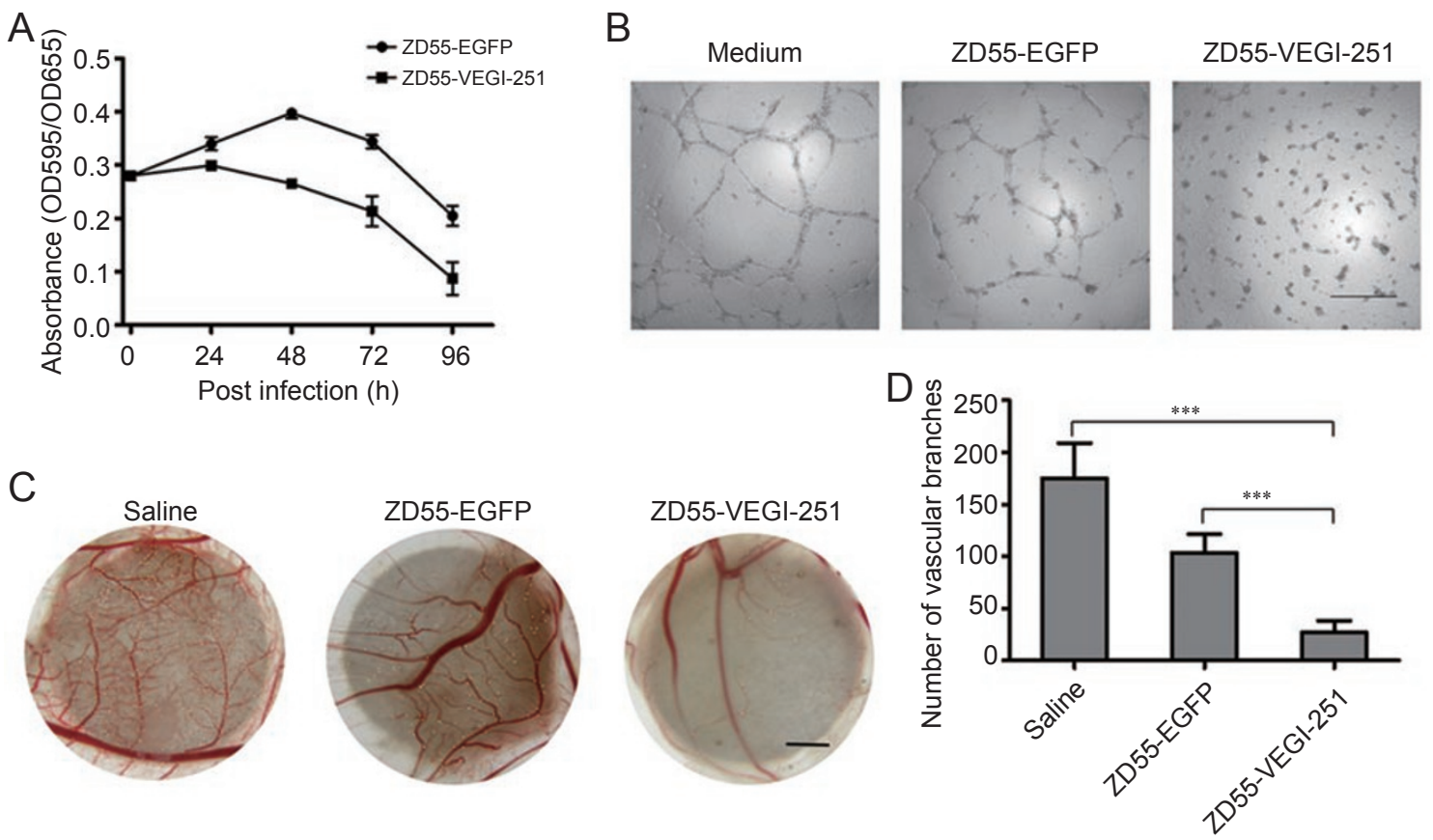

Figure 2 VEGI-251 inhibits the proliferation and tube formation of HUVECs in vitro and angiogenesis of CAM in vivo. (A) Measurement of the growth rate of HUVECs by MTT assay. Results were expressed as mean \pm SD values from five replicate wells of three separate experiments. (B) Tube formation of HUVECs on Matrigel was visualized by phase-contrast microscope. Scale bar, $500 \mu \mathrm{m}$. (C) Angiogenesis in CAM was photographed and the visible branches (indicated by yellow points) were counted. Scale bar, $1 \mathrm{~mm}$. (D) Statistical results of the vascular branches in CAM. Results were expressed as mean \pm $\mathrm{SD}(n=5, * * * P<0.001)$.

EGFP at a MOI of 10 and cell viability was measured by 3-(4,5-dimethylthiazol-2-yl)-2,5-diphenyltetrazolium bromide (MTT) assay. As shown in Figure 2A, ZD55VEGI-251 infection led to an about $70 \%$ decrease of the cell viability in $96 \mathrm{~h}$, whereas ZD55-EGFP infection caused only about $20 \%$ decrease. We then determined whether ZD55-VEGI-251 inhibits the ability of HUVECs to form capillary-like structures on a coating of extracellular proteins (Matrigel). At 6 hours after seeding HUVECs on Matrigel, the cells formed an extensive network of capillary-like tubes. When treated with CM of ZD55-VEGI-251-infected HeLa cells, the HUVECs no longer were able to form the capillary-like structures. In contrast, no significant morphological change was observed when HUVECs were incubated with $\mathrm{CM}$ of ZD55-EGFP-infected HeLa cells (Figure 2B).

\section{Inhibition of angiogenesis in chorioallantoic membrane (CAM)}

We further verified the in vivo antiangiogenic activity of the secreted VEGI-251 by using the CAM assay. In the control group, exposed areas of the CAM treated with saline exhibited abundant blood vessels and lots of branches (Figure 2C). Treatment with CM of ZD55-VE-
GI-251-infected HeLa cells for $48 \mathrm{~h}$ led to nearly complete inhibition of neovascularization on the CAM. CM of ZD55-EGFP-infected HeLa cells also affected CAM angiogenesis, but the effect was to a much less extent. Quantitative analysis was carried out by counting the number of vessel branches on the CAM of each group (five embryos each). The results indicated that treatment with CM of ZD55-EGFP- or ZD55-VEGI-251-infected HeLa cells caused $42 \%$ and $86 \%$ inhibition of the CAM angiogenesis, respectively (Figure 2D).

\section{ZD55-VEGI-251 selectively inhibits tumor cell prolifera- tion and induces cancer cell apoptosis}

It has been reported that only at a high concentration $(10 \mu \mathrm{g} / \mathrm{ml})$ and in the presence of cycloheximide, recombinant VEGI can activate caspase-3 and lead to PARP cleavage in human myeloid tumor cells U-937 [20]. Nevertheless, we examined the effect of ZD55-VEGI-251 on tumor cells to find out if the combination of VEGI251 and oncolytic virus generates better anticancer traits. Thus, human tumor cell lines HeLa, SMMC-7721, SW620 and human normal cell line L-02 were infected with ZD55-VEGI-251, ZD55-EGFP, ONYX-015 or AdVEGI-251 at different MOI, and the cytopathic effect 
A

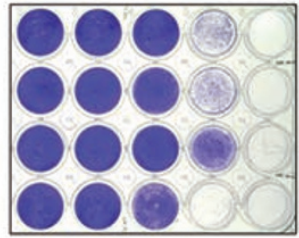

HeLa

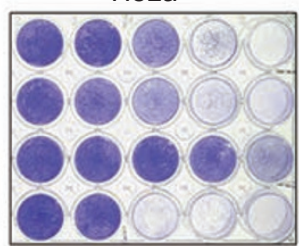

SW620

MOI $\begin{array}{lllll}0 & 0.1 & 1 & 10 & 100\end{array}$

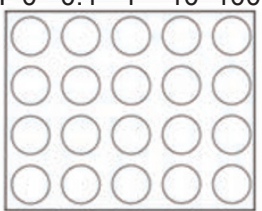

ONYX-015

ZD55-EGFP

Ad-VEGI-251

ZD55-VEGI-251

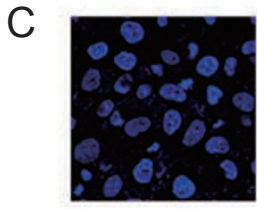

UI

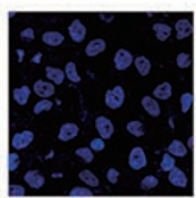

Ad-VEGI-251

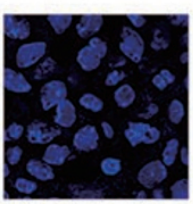

ONYX-015

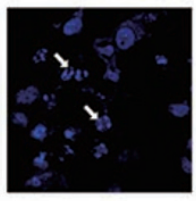

ZD55-VEGI-251

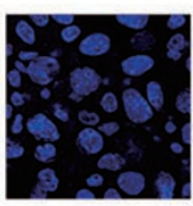

ZD55-EGFP

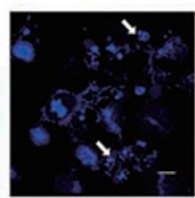

ZD55-TRAIL

B
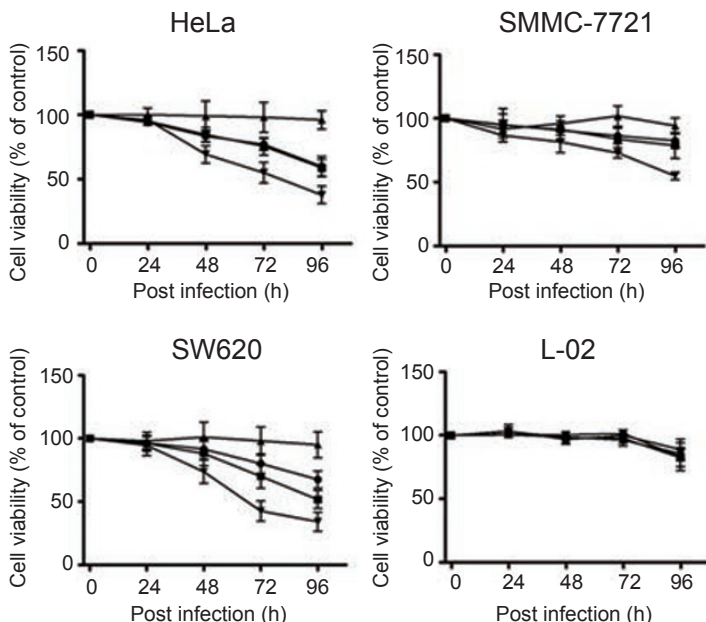

ONYX-015 -ZD55-EGFP —Ad-VEGI-251 -ZD55-VEGI-251

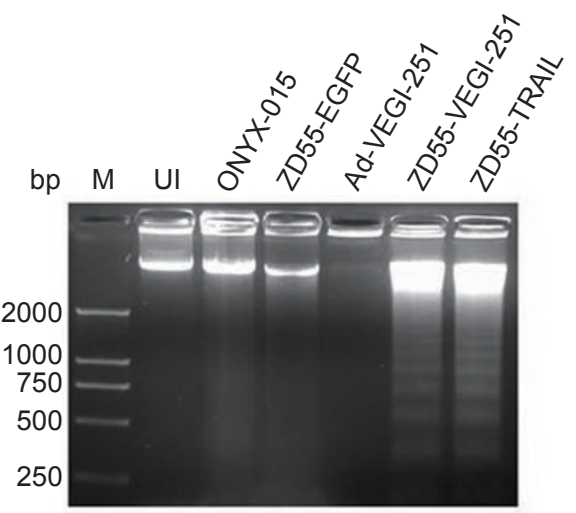

D
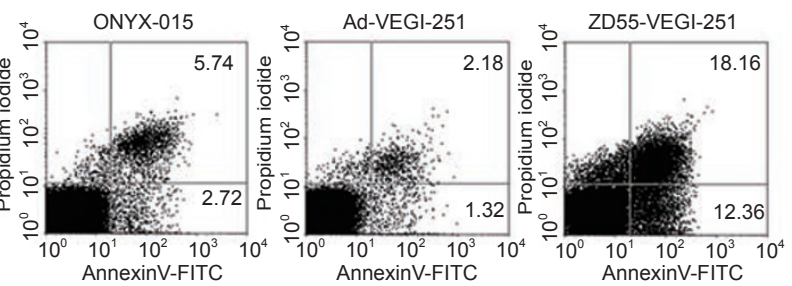

F

E

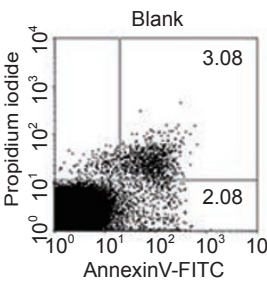

HeLa
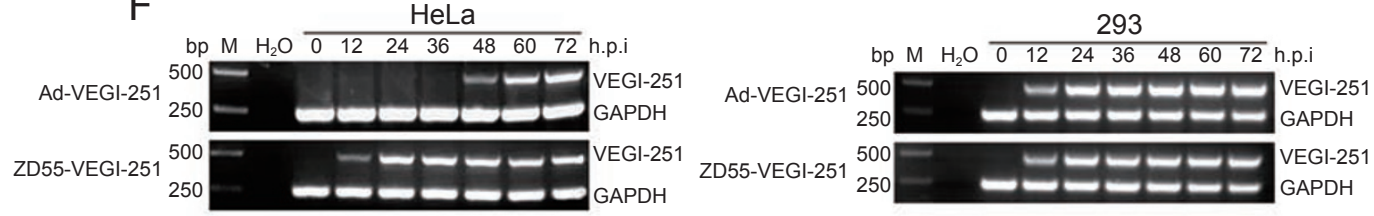

Figure 3 ZD55-VEGI-251 inhibits proliferation and induces apoptosis of tumor cells. (A) Cytopathic effect of different adenoviruses on HeLa, SMMC-7721, SW620 or L-02 cells at a series of MOI. (B) Measurement of cell viability of HeLa, SMMC7721 , SW620 or L-02 cells after infected with different adenoviruses at a MOI of 10 by MTT assay. Uninfected cells were considered as $100 \%$ viable. Points indicate mean values $(n=6)$; bars indicate SD. (C) The nuclear morphology of HeLa cells were visualized by a confocal microscope after stained with Hoechst33258 dye. The arrows are pointed toward the apoptotic cells. Scale bar, $10 \mu \mathrm{m}$. (D) DNA fragmentation assay of HeLa cells $48 \mathrm{~h}$ after being infected with different adenoviruses at a MOI of 10. M, DNA marker DL2000; UI, uninfected HeLa cells. (E) Detection of HeLa cells apoptosis by FACS after being stained with Annexin V-FITC and PI. Numbers show the percentage of cells in the first and fourth quadrants. (F) Detection of the mRNA level of VEGI-251 after virus infection in HeLa or 293 cells by RT-PCR. h.p.i., hours post-infection. GAPDH mRNA was used as an internal control. 
and cell viability were measured by crystal violet staining and MTT assay, respectively. As shown in Figure 3A, significantly stronger cytopathic effect was observed in all tumor cell lines infected with ZD55-VEGI-251, as compared with cells infected with ONYX-015, ZD55EGFP or Ad-VEGI-251. To further evaluate the kinetics of cytotoxicity induced by ZD55-VEGI-251, cells were plated in 96-well plates and infected with the four recombinant adenoviruses at a MOI of 10 , and subjected to viability measurement. The viability of tumor cells infected with ZD55-VEGI-251 was markedly lower than that of ONYX-015, ZD55-EGFP or Ad-VEGI-251, and the inhibition effect was time-dependent. Interestingly, no significant inhibition of cell proliferation was observed on normal cells infected with any of these viruses (Figure 3B). These results suggest that ZD55-VEGI-251 can selectively replicate in tumor cells and inhibit the growth of a wide variety of tumor cells more effectively than ONYX-015, ZD55-EGFP and Ad-VEGI-251.

To investigate whether apoptosis was involved in ZD55-VEGI-251-induced cytotoxicity, several assays for apoptosis detection were carried out. Similar to ZD55TRAIL, ZD55-VEGI-251 infection can apparently cause tumor cell nuclear fragmentation, chromatin condensation (Figure 3C) and DNA fragmentation (Figure 3D). In addition, tumor cells were subjected to annexin Vfluorescein isothiocyanate (FITC) and propidium iodide
(PI) staining, and flow cytometric analysis. As shown in Figure $3 \mathrm{E}$, the percentage of annexin V-positive population in ZD55-VEGI-251-infected tumor cells was found to be $30.52 \%$, as compared with $8.46 \%$ of ONYX- 015 infected and $5.16 \%$ of mock-infected cells. These results indicate that ZD55-VEGI-251 can efficiently eliminate tumor cells by inducing apoptosis. It was interesting to note that Ad-VEGI-251 was ineffective on tumor cells because it is replication-deficient due to the lack of both E1A and E1B genes. Another possibility is that VEGI251 expression in Ad-VEGI-251-infected tumor cells appeared to be attenuated, as VEGI was not detected until $48 \mathrm{~h}$ post-infection. In sharp comparison, VEGI-251 expression in ZD55-VEGI-251-infected cancer cells was initiated within $12 \mathrm{~h}$ post-infection, and could be maintained along with ZD55-VEGI-251 replication (Figure $3 \mathrm{~F}$, left). However, there was no difference of VEGI-251 expression between Ad-VEGI-251- and ZD55-VEGI251 -infected 293 cells, because 293 cells can express E1A and E1B proteins and confer the replication ability on Ad-VEGI-251 (Figure 3F, right).

\section{ZD55-VEGI-251 can activate mitochondria-mediated apoptosis signaling pathway}

In order to explain why ZD55-VEGI-251 but not ZD55-EGFP can induce apparent apoptosis in tumor cells and determine the underlying mechanism, several

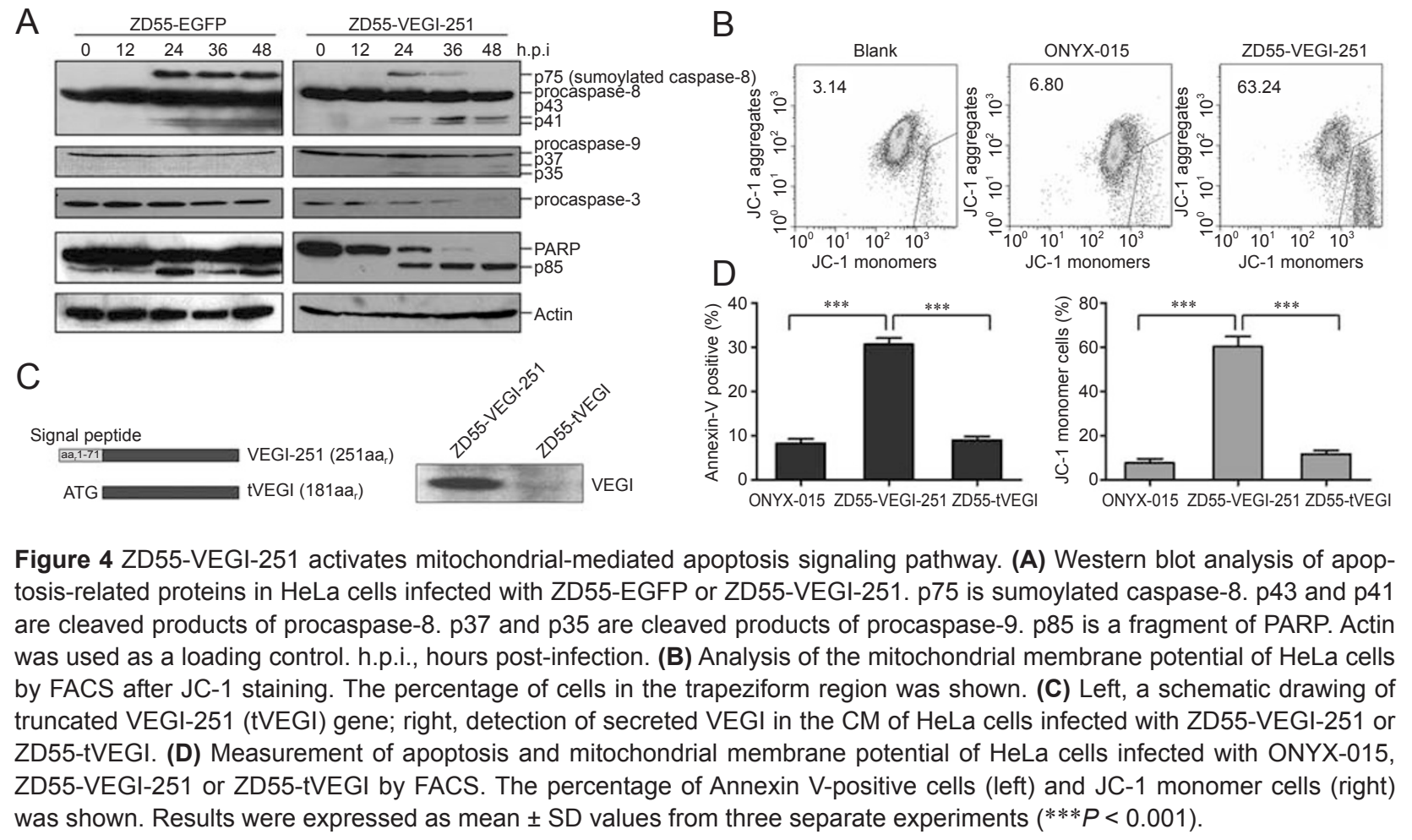

www.cell-research.com | Cell Research 

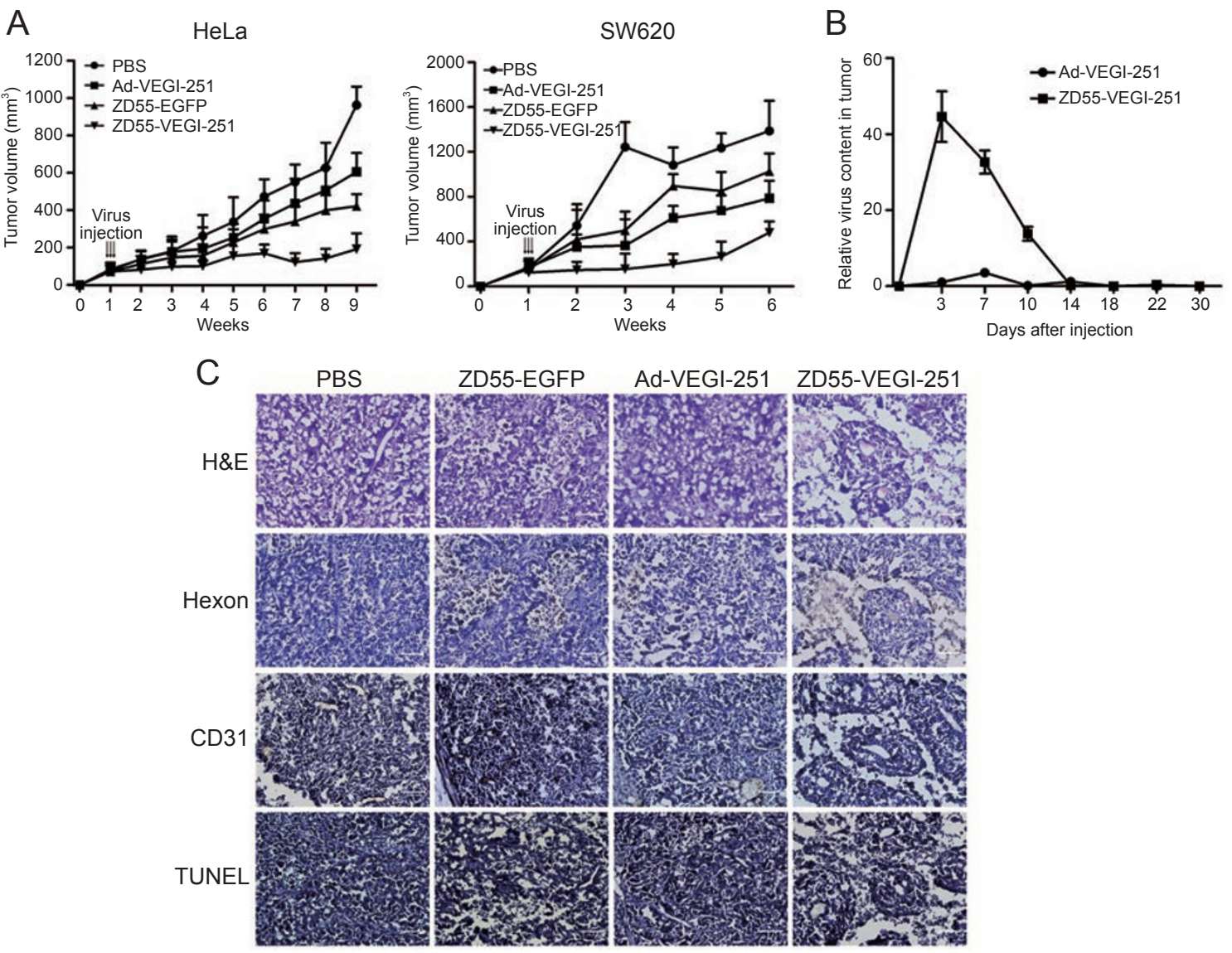

Figure 5 ZD55-VEGI-251 inhibits the growth of HeLa and SW620 xenograft tumors. (A) Antitumor effect of different adenoviral injections on HeLa and SW620 xenograft tumors. Points indicate mean values (HeLa, $n=6$; SW620, $n=8$ ); bars indicate SD. (B) Analysis of the viral kinetics of Ad-VEGI-251- or ZD55-VEGI-251-treated SW620 tumors by real-time PCR. Adenovirus E3 region was amplified to evaluate the viral DNA content. $\beta$-actin DNA was used as an internal control. (C) Analysis of tumor sections derived from PBS- or various adenoviruses-treated tumors by hematoxylin and eosin staining, hexon and CD31 immunostaining, TUNEL staining. Scale bar, $100 \mu \mathrm{m}$.

apoptosis-related proteins were analyzed by western blot (Figure 4A). It is interesting to note that caspase-8 can be sumoylated after virus infection, which is associated with its nuclear localization [21]. The difference was that sumoylated caspase-8 seemed stable in ZD55EGFP-infected cells, but gradually disappeared over time in ZD55-VEGI-251-infected cells. As poly(ADPribose) polymerase-2 (PARP-2) cleavage associates with the disappearance of the $\mathrm{p} 75$ form of caspase- 8 in the nuclear fractions [21] and procaspase- 3 is a substrate of active caspase-8 [22], enhanced PARP cleavage and procaspase-3 activation were observed in ZD55-VEGI251-infected cells. Moreover, only ZD55-VEGI-251 can induce cleavage of caspase-9, suggesting the activation of mitochondria-mediated apoptosis signaling pathway. To corroborate this finding, we determined mitochondrial transmembrane electrical potential $(\Delta \Psi)$ by JC-1 stain- ing. Fluorescence-activated cell-sorting (FACS) analysis revealed that ZD55-VEGI-251 infection gave rise to a significant descent of mitochondrial transmembrane electrical potential (Figure 4B). These observations indicate that VEGI-251 effectively enhanced ZD55-induced apoptosis by modulating caspase- 8 and the mitochondrial pathway.

Secretion of VEGI-251 is necessary for ZD55-VEGI-251induced cell apoptosis

Since VEGI-251 is a secreted protein, we investigated whether secretion of VEGI-251 by ZD55-VEGI-251infected cells was important for induction of the apoptosis cascade. A non-secretable VEGI was constructed by deleting the secretion signal peptide from the $\mathrm{N}$-terminus (Figure 4C), and then the truncated gene was inserted into the vector ZD55 to construct ZD55-tVEGI. Dramati- 
cally, when used to infect the tumor cells, ZD55-tVEGI could neither induce apparent cell apoptosis nor change the mitochondrial transmembrane electrical potential obviously, which is similar to the action of ONYX-015 (Figure 4D). The results indicate that secretion of VEGI251 is necessary in the process of ZD55-VEGI-251-induced tumor cell apoptosis. However, it is surprising that the known VEGI receptor DR3 was not detected in HeLa cells (Supplementary information, Figure S3), suggesting that there is an unknown VEGI membrane receptor in HeLa cells.

Intratumoral injection of ZD55-VEGI-251 suppresses tumor growth

A model of HeLa human cervical tumor or SW620 human colorectal tumor xenografts was established on athymic nude mice to investigate the antitumor efficacy of ZD55-VEGI-251 in vivo. As shown in Figure 5A, various degrees of tumor growth inhibition were observed after intratumoral injection of different viruses. The average volume of ZD55-VEGI-251-treated HeLa tumors was about $180 \mathrm{~mm}^{3}$ at the end of the experiment compared with the average tumor volume of $950 \mathrm{~mm} 3$ in the untreated group. This represents an $80 \%$ suppression of the xenograft tumor growth. Similar results were also observed in the SW620 xenograft tumor model. In both animal models, ZD55-VEGI-251 exhibited much more potent antitumor efficacy as compared with ZD55EGFP because of the expression of VEGI-251. The effect of Ad-VEGI-251 was poor because of its replication deficiency. Analysis of the viral kinetics in vivo further demonstrated the advantages of ZD55-VEGI-251. At 3 days after injection, the viral replication of ZD55-VEGI-251 reached peak value, which was almost 50 times the amount of Ad-VEGI-251 and could last for 2 weeks. In contrast, Ad-VEGI-251 was difficult to detect a week after injection (Figure 5B). The result of tracing EGFP expression in vivo also supported this finding (Supplementary information, Figure S4).

Tumor specimens were obtained from tumor-bearing animals 1 week after treatment and analyzed for the extent of viral production, neovascularization and apoptosis (Figure 5C and Supplementary information, Figure S5). $\mathrm{H}$ and $\mathrm{E}$ staining revealed large areas of necrotic tissue

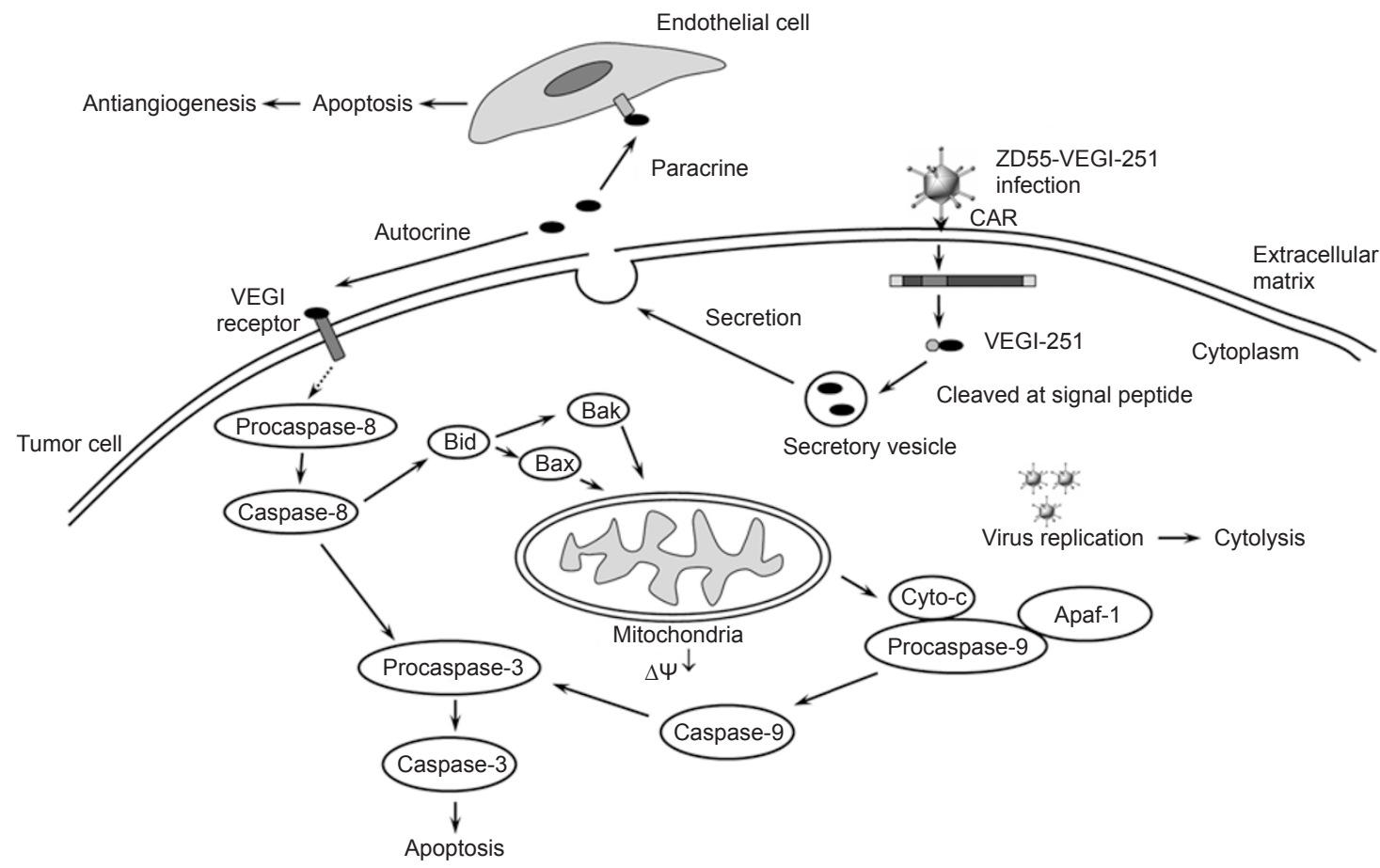

Figure 6 Possible mechanisms of the antitumor effect of ZD55-VEGI-251 in vivo. After ZD55-VEGI-251 infection, on the one hand, VEGI-251 can be immediately expressed and cleaved at signal peptide, and then secreted to the extracellular environment. In paracrine pathway, VEGI can induce endothelial cells apoptosis and inhibit angiogenesis. In autocrine pathway, VEGI binds to its receptor on tumor cell surface, resulting in activation of caspase- 8 and caspase- 9 . Then, the downstream executioner caspase- 3 is activated and cleaves proteins involved in programmed cell death events such as PARP. On the other hand, replication of ZD55-VEGI-251 can cause cytolytic effect on tumor cells. These potent functions confer ZD55VEGI-251 a promising candidate for cancer therapy. 
after ZD55-VEGI-251 treatment that matched the areas stained brown by anti-hexon antibody, indicating that ZD55-VEGI-251 can effectively cause tumor necrosis in vivo. CD31 immunostaining was used to evaluate the effect of ZD55-VEGI-251 on tumor angiogenesis. The microvessel density in tumors derived from the ZD55-VEGI-251-treated group was markedly reduced compared with that of other groups. TUNEL analysis revealed that injection of ZD55-VEGI-251 caused profound apoptosis in the tumors, while no apoptosis was seen in PBStreated tumors. ZD55-EGFP infection also led to some apoptosis because of virus replication, but the percentage of apoptotic cells was much smaller than that in ZD55VEGI-251-treated tumors. On the basis of the above observations, we propose that the antitumor activity of ZD55-VEGI-251 arises from a combination of three effects: the oncolytic ability of ZD55, the apoptosis-inducing activity due to the autocrine action of VEGI-251 and the antiangiogenic effect due to the paracrine action of VEGI-251 (Figure 6).

\section{Lack of general toxicity of the ZD55-VEGI-251 viral preparation}

To further assess the safety of the adenoviral preparations applied in the animal experiments, the activities of three enzymes critical to liver function: aspartate aminotransferase (AST), alanine aminotransferase (ALT) and alkaline phosphatase (ALP), in the sera of the experimental animals were measured (Table 1). Compared with the data obtained from PBS-treated animals, no significant change of any the indexes was observed in the groups that received intratumoral injections of ZD55-EGFP, AdVEGI-251 or ZD55-VEGI-251. This initial assessment of toxicity suggests a promising potential for application of ZD55-VEGI-251 in clinical settings.

\section{Discussion}

The anticancer activity of VEGI has been mostly ascribed to its ability to inhibit neovascularization by inducing apoptosis in proliferating endothelial cells $[10$,
$23]$, and to activate $\mathrm{T}$ cells, natural killer cells and dendritic cells $[13,24]$. These activities rely on an autocrine mechanism for the largely endothelial cell-produced cytokines to act on endothelial cells themselves, and a paracrine mechanism to act on the immune cells. In this study, with the original aim of achieving a combination of antiangiogenic gene therapy and oncolytic viral therapy, a secretable form of the cytokine (VEGI-251) was chosen as the therapeutic gene.

We constructed an E1B-attenuated oncolytic adenovirus, ZD55-VEGI-251, by inserting a VEGI-251 expression cassette into an engineered ZD55-gene system, which has been shown to be a potent antitumor strategy in our previous studies [16-18, 25]. As anticipated, on ZD55-VEGI-251 infection, VEGI-251 is immediately expressed and released to the extracellular environment of the host cells to exert its function as an antiangiogenic cytokine. Using CM of HeLa cells infected with ZD55VEGI-251, potent inhibition of proliferation and capillary-like tube formation of HUVECs as well as inhibition of CAM angiogenesis were observed. Intriguingly, in the process of collecting the $\mathrm{CM}$, we found that the cancer cells infected with ZD55-VEGI-251 were very different morphologically from those infected with ZD55-EGFP, with the former obviously exhibiting characteristics of apoptotic cells. This finding prompted us to investigate whether VEGI-251 can enhance the oncolytic effect of ZD55 through an autocrine mechanism. Indeed, we fortuitously discovered that ZD55-VEGI-251 can activate the mitochondria-mediated apoptosis signaling pathway and induce caspase-dependent cancer cell apoptosis. In addition, it was demonstrated that the secretion of VEGI-251 is a prerequisite for induction of the apoptosis cascade. Furthermore, intratumoral injection of ZD55-VEGI-251 gave rise to a remarkable suppression of the growth of xenograft tumors. These findings together strongly support the view that ZD55-VEGI-251 can not only inhibit angiogenesis via the paracrine action of VEGI-251 but also directly induce apoptosis of the host cancer cells via virus replication and the autocrine action of VEGI-251.

Several studies on VEGI gene function using recom-

Table 1 Indexes of serum liver function enzymes 3 days after intratumoral injection of adenovirus in nude mice

\begin{tabular}{llcccc}
\hline & PBS $^{1}$ & ONYX-015 & ZD55-EGFP & Ad-VEGI-251 & ZD55-VEGI-251 \\
\hline ALT & $61 \pm 7^{2}$ & $64 \pm 12$ & $49 \pm 5$ & $62 \pm 14$ & $52 \pm 6$ \\
AST & $203 \pm 55$ & $218 \pm 33$ & $170 \pm 24$ & $228 \pm 36$ & $181 \pm 22$ \\
ALP & $139 \pm 14$ & $131 \pm 16$ & $125 \pm 7$ & $123 \pm 18$ & $123 \pm 9$ \\
AST/ALT & $3.4 \pm 1.1$ & $3.5 \pm 0.5$ & $3.5 \pm 0.5$ & $3.8 \pm 0.6$ & $3.5 \pm 0.6$ \\
\hline
\end{tabular}

${ }^{1}$ Each group was composed of six nude mice.

${ }^{2}$ All data were expressed as the mean $\pm \mathrm{SD}$ and the unit of serum enzymes is international units per liter (IU/L). 
binant protein or on stable cell lines expressing secreted VEGI have demonstrated the ability of this unique cytokine to inhibit tumor growth in animal models, but clinical application of VEGI has not yet been achieved $[10,26]$. In this study, we show that because expression of VEGI-251 is immediate after virus infection and can escalate along with virus replication, the oncolytic adenovirus ZD55-VEGI-251 is advantageous compared with the replication-deficient adenovirus Ad-VEGI-251. In addition to the difference in VEGI-251 expression between ZD55-VEGI-251- and Ad-VEGI-251-infected cells, we shall also consider the function of the viral proteins. ZD55-VEGI-251 only fails to produce the E1B $55 \mathrm{kDa}$ protein, while Ad-VEGI-251 lacks the entire E1A and E1B genes. Thus, distinguishing from Ad-VEGI-251, ZD55-VEGI-251 can still express E1A and E1B $19 \mathrm{kDa}$ proteins. While E1A is shown to be sufficient by itself to induce apoptosis of host cells independently of p53, cell death is significantly prevented when E1B $19 \mathrm{kDa}$ is co-expressed [27]. It is therefore plausible that ZD55VEGI-251-induced cancer cell apoptosis results from the combined effect of E1A and VEGI-251 proteins.

Our previous studies revealed that the antitumor effect of an individual ZD55-gene virus is often limited [18, $25]$. Because the genomes of tumor cells are invariably altered at multiple sites and several important signaling pathways are always disrupted at the same time [28], a combination of genes that target totally different aspects of tumor biology would achieve more potent inhibition in a wide variety of tumors. Indeed, we are currently attempting to construct an oncolytic adenovirus expressing both VEGI-251 and manganese superoxide dismutase. Moreover, chemotherapy and radiotherapy could be combined with the ZD55-gene system to complement its antitumor activity $[18,29]$.

While considering how the factors embodied in a therapeutic approach such as an oncolytic virus may work together to maximize clinical benefits [30], it is equally important to take into account some undesired side effects. In this study, we have determined the biochemical functions of liver enzymes AST, ALT and ALP in the sera of the experimental animals, and found the absence of hepatotoxicity. However, it should be pointed out that only intratumoral injection of ZD55-VEGI-251 was used here to treat local xenograft tumors. The safety of ZD55VEGI-251 needs to be further evaluated with the virus being administered systemically, especially if it is to be used to treat cancer metastasis.

In conclusion, we showed here that secreted VEGI251 from ZD55-VEGI-251-infected cells can inhibit endothelial cell proliferation in vitro and angiogenesis in vivo. In addition, we present a novel finding that ZD55-
VEGI-251 infection can lead to the activation of the mitochondrial apoptosis signaling pathway. Importantly, the induction of host cell apoptosis depends on VEGI being secreted by the host cells. These results suggest the possibility of taking advantage of the apoptotic effect and the antiangiogenic ability of ZD55-VEGI-251 for clinical cancer therapy.

\section{Materials and Methods}

\section{Cell lines and culture conditions}

Human cervical cancer cell line HeLa, human colorectal cancer cell line SW620, human hepatoma cell line SMMC-7721 and normal human liver cell line L-02 were purchased from the Cell Bank of Type Culture Collection of Chinese Academy of Sciences (Shanghai, China). HEK293 was obtained from Microbix Biosystems Inc. (Toronto, Ontario, Canada). HUVECs were isolated from freshly obtained human umbilical cords following Jaffe's methods [31] and cultured in M199 (GIBCO BRL, Grand Island, NY, USA) containing 20\% fetal bovine serum (FBS, GIBCO BRL) and 75 $\mu \mathrm{g} / \mathrm{ml}$ endothelial cell growth supplement (Sigma, St Louis, MO, USA). Flow cytometric analysis indicates that nearly $100 \%$ of the cells were CD31 (PECAM-1)-positive (Supplementary information, Figure S2). HEK293 cell line was cultured in Dulbecco's modified Eagle's medium (DMEM; GIBCO BRL) supplemented with $10 \%$ heat-inactivated FBS. The L-02 cell line was grown in RPMI 1640 (GIBCO BRL) supplemented with 5\% FBS. Other cell lines were cultured in DMEM supplemented with 5\% FBS. All cells were incubated at $37{ }^{\circ} \mathrm{C}$ in a humidified air atmosphere with $5 \% \mathrm{CO}_{2}$.

\section{Plasmids}

Adenoviral shuttle vector pCA13 and adenoviral packaging vector $\mathrm{pBHGE} 3$ were obtained from Microbix Biosystems. pZD55 was constructed by our group previously [19]. The VEGI-251 gene (digested with $E c o \mathrm{RI} / X h o \mathrm{I}$ ) from pcDNA3-VEGI-251 was subcloned into the EcoRI and XhoI sites of pCA13 to construct pCA13-VEGI-251. Truncated VEGI-251 (tVEGI) with the deletion of 4-213 bp of VEGI-251 was amplified by PCR using forward (5'-CGG AAT TCA TGC TAA AAG GAC AGG AGT TTG CAC CTT C-3') and reverse (5'-CGG GAT CCT ATA GTA AGA AGG CTC CAA AGA AGG TTT TAT C-3') primers. The PCR products were digested with $E c o R \mathrm{I}$ and $\mathrm{BamHI}$, and then cloned into pCA13 to construct pCA13-tVEGI. The pZD55-VEGI-251 (or pZD55-tVEGI) was constructed by inserting the whole VEGI-251 (or tVEGI) expression cassette derived from pCA13-VEGI-251 (or pCA13-tVEGI) using BglII into the corresponding site of pZD55. Adenovirus E1A proteins expressing plasmid pcDNA3-E1A was kindly provided by Professor Yipeng Qi (Laboratory of Molecular Virology, College of Life Sciences, Wuhan University, Wuhan, Hubei Province, China). All plasmid constructs were confirmed by restriction enzyme digestion, PCR and DNA sequencing.

Generation, identification, purification and titration of adenovirus

Ad-VEGI-251, ZD55-VEGI-251 and ZD55-tVEGI were generated by homologous recombination of pCA13-VEGI-251, pZD55VEGI-251 and pZD55-tVEGI with adenoviral packaging vector 
pBHGE3 in HEK293 cells. Individual plaques were picked up and used to infect HEK293 cells. After observing apparent cytopathic effect, the cell culture medium was collected and virus genomic DNA were extracted. Then, the contamination of wild-type adenovirus and the presence of foreign gene expression cassettes were identified by PCR using primer pairs targeting E1B region or exogenous gene (Supplementary information, Figure S1). Primers for virus identification are listed in Supplementary information, Table S1. Recombinant adenoviruses were amplified by infecting HEK293 cells and purified by cesium chloride gradient ultracentrifugation. The titration of virus was determined by standard plaque assay using HEK293 cells. ONYX-015, ZD55-EGFP, ZD55-TRAIL and wild-type adenovirus were preserved in our lab.

\section{Cytopathic assay}

HeLa, SMMC-7721 and SW620 tumor cell lines, as well as L-02 normal cell line were grown to subconfluent levels in a 24well plate (Corning, NY, USA) and infected with ONYX-015, ZD55-EGFP, Ad-VEGI-251 or ZD55-VEGI-251 at various MOIs. At 7 days after infection, the medium was removed and cells were exposed to $2 \%$ crystal violet in $20 \%$ methanol for $15 \mathrm{~min}$. Then plates were washed with distilled water $\left(\mathrm{dH}_{2} \mathrm{O}\right)$ and documented as photographs.

\section{Cell viability assay}

Cells were plated on 96-well plates (Corning) at a density of 6 $\times 10^{4}$ cells per well. When cells were grown to subconfluent levels, they were infected with ONYX-015, ZD55-EGFP, Ad-VEGI-251 or ZD55-VEGI-251 at a MOI of 10. At different time points (24, 48, 72 and $96 \mathrm{~h}$ ), $20 \mu \mathrm{l}$ of MTT (Sigma) solution $(5 \mathrm{mg} / \mathrm{ml})$ was added into each well. Cells were incubated at $37{ }^{\circ} \mathrm{C}$ for $4 \mathrm{~h}$. Then the medium was removed and $100 \mu \mathrm{l}$ of $0.04 \mathrm{M} \mathrm{HCl}$-isopropanol solution was added to each well to solubilize the formazan crystals. Absorbance was measured at dual wavelength mode $(595 \mathrm{~nm}$ and $655 \mathrm{~nm}$ ) using a Microplate Reader (Bio-Rad, Hercules, CA, USA). The percentage of cell viability was calculated as follows: (mean value $A_{595 / 655}$ of infected cells)/(mean value $A_{595 / 655}$ of uninfected cells) $\times 100 \%$.

\section{Hoechst33258 staining and DNA fragmentation assay}

HeLa cells grew on glass coverslips in a six-well plate and were treated with ONYX-015, ZD55-EGFP, Ad-VEGI-251 or ZD55VEGI-251 at a MOI of 10 for $48 \mathrm{~h}$ or untreated (mock). Cells were fixed with 4\% paraformaldehyde and stained with Hoechst 33258 (Molecular Probes, Eugene, OR, USA) at $1 \mu \mathrm{g} / \mathrm{ml}$ for $10 \mathrm{~min}$. Then, cells were washed with PBS twice and visualized with a Leica TCS SP2 confocal microscope (Leica Microsystems, Wetzlar, Germany). For DNA fragmentation assay, HeLa cells were seeded in 10 -cm culture dishes $\left(5 \times 10^{6}\right.$ cells per dish $)$ and infected with these viruses. At $72 \mathrm{~h}$ post-infection, DNA were extracted according to Herrmann's method [32] and analyzed using agarose gel electrophoresis.

\section{Flow cytometric analysis}

For apoptosis detection, both adherent and floating cells were collected $48 \mathrm{~h}$ after adenovirus infection $(\mathrm{MOI}=10)$ and washed twice with PBS. Then, cells were resuspended in $500 \mu \mathrm{l}$ binding buffer and stained with FITC-labeled Annexin V and PI (BioVision, Palo Alto, CA, USA) according to the manufacturer's instructions.
For analysis of mitochondrial membrane potential, cells were harvested from experimental samples and stained with $2.5 \mu \mathrm{g} / \mathrm{ml} \mathrm{JC}-1$ fluorescent probe (Sigma) in a dark place at room temperature for 20 min. Then, cells were washed twice with PBS and were made ready for analysis. For identification of primary cultured HUVECs, second-generation HUVECs were trypsinized and washed once with complete medium. Then, cells were incubated with mouse anti-human CD31-FITC monoclonal antibody (AbD Serotec, Kidlington, Oxford, UK) at room temperature for 30 min avoiding light and washed with PBS twice. A FACS assay was performed immediately after staining in a FACSCalibur flow cytometer (BD Biosciences, Franklin Lakes, NJ, USA).

\section{RT-PCR analysis}

Total RNA was isolated from human cell lines using Trizol reagent (Invitrogen, Carlsbad, CA, USA). Single-strand cDNA for a PCR template was synthesized from $10 \mu \mathrm{g}$ of total RNA using Oligo(dT $)_{18}$ primers and RevertAid First Strand cDNA Synthesis Kit (Fermentas, Vilnius, Lithuania). The oligonucleotide primers used for amplification were listed in Supplementary information, Table S1. Equal amounts of each reverse transcription product $(1 \mu \mathrm{l})$ were amplified using Taq polymerase. The PCR products were run on $1.5 \%$ agarose gels and visualized by ethidium bromide.

\section{Western blot analysis}

Cells were harvested from the plates and resuspended in lysis buffer. Protein concentrations were determined with the BioRad protein assay system and western blot were carried out using standard procedures. Caspase- 8 and caspase- 9 antibodies were purchased from Cell Signaling Inc. (Danvers, MA, USA). VEGI, caspase-3, poly (ADP-ribose) polymerase (PARP), actin antibodies and horseradish peroxidase-conjugated secondary antibodies were obtained from Santa Cruz Biotechnology (Santa Cruz, CA, USA).

\section{Tube formation assay}

The 96-well cell culture plates were coated with $100 \mu \mathrm{l}$ per well of Matrigel (BD Biosciences, Bedford, MA, USA) and incubated at $37{ }^{\circ} \mathrm{C}$ for $30 \mathrm{~min}$ to promote gelling. HUVECs (second to third passages) were resuspended at $1.3 \times 10^{5}$ cells per $\mathrm{ml}$ in M199 medium supplemented with 2\% heat-inactivated FBS after serum starvation for $12 \mathrm{~h}$. Aliquots of cells $(100 \mu \mathrm{l}$ per aliquot) were seeded to each Matrigel-containing well. CM of ONYX015, ZD55-EGFP, Ad-VEGI-251 or ZD55-VEGI-251-infected HeLa cells were concentrated using Amicon Ultra-4 centrifugal filter devices (Millipore, Billerica, MA, USA) and $100 \mu \mathrm{l}$ of each concentrated sample were added into the wells. Complete medium was used as untreated control. After $6 \mathrm{~h}$, the tube structures were identified and photographed. Three replicate wells were tested per sample.

\section{CAM assay}

Fertilized chick embryos were incubated for 10 days at $37{ }^{\circ} \mathrm{C}$ with $70 \%$ humidity. A small hole was drilled into the air sac and then the embryos were candled to determine a location to drill a second hole directly over embryonic blood vessels. The CAM was separated from the egg's shell by pressing an evacuated Pasteur pipette bulb against the first (air sac) hole. A small window (10-15 mm in diameter) was created in the egg's shell over the dropped CAM with a forceps, exposing the CAM for subsequent 
experimental manipulation. Cortisone acetate-treated filter disks (6 $\mathrm{mm}$ in diameter) were soaked with $10 \mu \mathrm{l}$ of concentrated CMs of ONYX-015, ZD55-EGFP, ZD55-VEGI-251-infected HeLa cells or normal saline and placed directly onto an avascular area containing a pre-existing blood vessel of CAM. The windows were sealed with transparent tape and incubated at $37^{\circ} \mathrm{C}$. After $72 \mathrm{~h}$, eggs were transferred to ice for $30 \mathrm{~min}$ and the filters were removed by cutting the CAM around the perimeter of the filter disk, leaving a margin of $5 \mathrm{~mm}$. The filters plus CAM were rinsed and inverted to reveal the tissue below the filter. After photographing, the quantification of angiogenesis was carried out by branch counting.

\section{Animal experiments}

Animal experiments were performed according to the Guide for the Care and Use of Laboratory Animals. Female BALB/c nude mice (4-week old) were purchased from Shanghai Experimental Animal Center (Shanghai, China). For establishment of xenograft tumors, $5 \times 10^{6} \mathrm{HeLa}$ or SW620 cells in $150 \mu 1$ DMEM were subcutaneously injected into the right flank of each mouse. When tumors reached $100-150 \mathrm{~mm}^{3}$ in size, mice were divided randomly into five groups (eight mice per group). Adenoviruses $\left(2 \times 10^{9}\right.$ PFUs per mouse) or PBS was injected into the tumors every other day for four times. The tumor volume $\left(\mathrm{mm}^{3}\right)$ was measured by a vernier caliper twice a week and calculated as (length $\times$ width $\left.^{2}\right) / 2$. For analysis of the indexes of serum liver function enzymes, the mice blood were collected from the tail vein 3 days after intratumoral injection of adenovirus and then AST, ALT and ALP in mice serum were measured by the ratio method. For tracing the EGFP expression in vivo, tumors injected with ZD55-EGFP were collected at 3, 7, 14, 21 and 30 days after injection. Tumor tissues were fixed in $4 \%$ formaldehyde and cut into $10-\mu \mathrm{m}$-thick sections. After staining with 4'-6-diamidino-2-phenylindole (DAPI), sections were visualized with an Olympus BX51 microscope (Olympus, Tokyo, Japan). For studying viral kinetics in vivo, tumors injected with Ad-VEGI-251 or ZD55-VEGI-251 were collected at 3, 7, 10, 14, 18, 22 and 30 days after injection and quickly frozen in liquid nitrogen. Then, total tissue DNA were extracted by Genomic DNA MiniPreps Kit (Generay Biotech, Shanghai, China) and viral DNA contents were measured by real-time PCR using adenovirus E3 region primers. Genomic DNA amplified by $\beta$-actin primers were used as an internal control.

\section{Immunohistochemistry}

Tumor tissues were fixed in 4\% formaldehyde and embedded in Jung tissue freezing medium (Leica Microsystems, Nussloch Gmbh, Nussloch, Germany). Then, 8- $\mu$ m-thick sections were cut from the frozen pituitary blocks with a Leica CM 3050S freezing microtome, thaw-mounted on gelatin-coated microscope slides and air-dried. The frozen sections were washed with PBS twice and endogenous peroxidase activity was eliminated by $30-\mathrm{min}$ incubation in $1 \% \mathrm{H}_{2} \mathrm{O}_{2}$ and $0.4 \%$ Triton $\mathrm{X}-100$ in PBS. Then the sections were blocked with the blocking serum and incubation with rat anti-mouse CD31 monoclonal antibody (1:200 dilution; BD Biosciences) or goat anti-adenovirus polyclonal antibody (1:200 dilution; Millipore) for $1 \mathrm{~h}$ at room temperature. The following procedures were performed using rat or goat ABC Staining System (Santa Cruz Biotechnology) according to the manufacturer's instructions and hematoxylin was used as a counterstain. All sections were visualized with a Nikon Eclipse 80i microscope (Nikon,
Tokyo, Japan).

Terminal deoxynucleotidyl transferase-mediated dUTPbiotin nick end labeling assay

Apoptotic cells in tumor tissue sections were assessed by terminal deoxynucleotidyl transferase-mediated dUTP-biotin nick end labeling staining with a TACS TdT Kit In Situ Apoptosis Detection Kit (R\&D, Minneapolis, MN, USA) according to the manufacturer's protocol. All sections were counterstained with hematoxylin and visualized with a Nikon Eclipse $80 \mathrm{i}$ microscope.

\section{Statistical analysis}

All data were expressed as the mean \pm SD and were analyzed using independent samples $t$-test and one-way analysis of variance (ANOVA) by SPSS Base 10.0. Results were considered statistically significant when $P<0.05$.

\section{Acknowledgments}

We thank Lanying Sun, Yang Xiao, Yuelei Chen, Hua Zhou and Cell Analysis Center (Institute of Biochemistry and Cell Biology, Shanghai Institutes for Biological Sciences, Chinese Academy of Sciences) for professional technical assistance. This work was supported in part by grants from Hi-Tech Research Development Program of China (863 Program, No. 2007AA021006); the Key Project of the Chinese Academy of Sciences (No. KSCX2-YWR-09); the 973 Project (No. 2004CB518804); Grant 30623003 from National Nature Science Foundation of China and Grant 06DZ22032 from Science and Technology Commission of Shanghai Municipality.

\section{References}

1 Folkman J. Tumor angiogenesis: therapeutic implications. $N$ Engl J Med 1971; 285:1182-1186.

2 Folkman J, D'Amore PA. Blood vessel formation: what is its molecular basis? Cell 1996; 87:1153-1155.

3 Folkman J. Angiogenesis in cancer, vascular, rheumatoid and other disease. Nat Med 1995; 1:27-31.

4 Folkman J. Seminars in Medicine of the Beth Israel Hospital, Boston. Clinical applications of research on angiogenesis. $N$ Engl J Med 1995; 333:1757-1763.

$5 \mathrm{Wu}$ Y, Zhong Z, Huber J, et al. Anti-vascular endothelial growth factor receptor-1 antagonist antibody as a therapeutic agent for cancer. Clin Cancer Res 2006; 12:6573-6584.

6 Zhai Y, Ni J, Jiang GW, et al. VEGI, a novel cytokine of the tumor necrosis factor family, is an angiogenesis inhibitor that suppresses the growth of colon carcinomas in vivo. FASEB $J$ 1999; 13:181-189.

7 Yu J, Tian S, Metheny-Barlow L, et al. Modulation of endothelial cell growth arrest and apoptosis by vascular endothelial growth inhibitor. Circ Res 2001; 89:1161-1167.

8 Zhai Y, Yu J, Iruela-Arispe L, et al. Inhibition of angiogenesis and breast cancer xenograft tumor growth by VEGI, a novel cytokine of the TNF superfamily. Int J Cancer 1999; 82:131136.

9 Metheny-Barlow LJ, Li LY. Vascular endothelial growth inhibitor (VEGI), an endogenous negative regulator of angiogenesis. Semin Ophthalmol 2006; 21:49-58. 
10 Chew LJ, Pan H, Yu J, et al. A novel secreted splice variant of vascular endothelial cell growth inhibitor. FASEB J 2002; 16:742-744.

11 Parr C, Gan CH, Watkins G, Jiang WG. Reduced vascular endothelial growth inhibitor (VEGI) expression is associated with poor prognosis in breast cancer patients. Angiogenesis 2006; 9:73-81.

12 Prehn JL, Thomas LS, Landers CJ, et al. The T cell costimulator TL1A is induced by FcgammaR signaling in human monocytes and dendritic cells. J Immunol 2007; 178:40334038.

13 Tian F, Grimaldo S, Fujita M, et al. The endothelial cellproduced antiangiogenic cytokine vascular endothelial growth inhibitor induces dendritic cell maturation. J Immunol 2007; 179:3742-3751.

14 Kakuta Y, Kinouchi Y, Negoro K, Takahashi S, Shimosegawa T. Association study of TNFSF15 polymorphisms in Japanese patients with inflammatory bowel disease. Gut 2006; 55:15271528.

15 Picornell Y, Mei L, Taylor K, et al. TNFSF15 is an ethnicspecific IBD gene. Inflamm Bowel Dis 2007; 13:1333-1338.

$16 \mathrm{Chu} \mathrm{L}, \mathrm{Gu}$ J, Sun L, et al. Oncolytic adenovirus-mediated shRNA against Apollon inhibits tumor cell growth and enhances antitumor effect of 5-fluorouracil. Gene Ther 2008; 15:484-494.

17 Pei Z, Chu L, Zou W, et al. An oncolytic adenoviral vector of Smac increases antitumor activity of TRAIL against HCC in human cells and in mice. Hepatology 2004; 39:1371-1381.

18 Zhang Y, Gu J, Zhao L, et al. Complete elimination of colorectal tumor xenograft by combined manganese superoxide dismutase with tumor necrosis factor-related apoptosis-inducing ligand gene virotherapy. Cancer Res 2006; 66:4291-4298.

19 Zhang ZL, Zou WG, Luo CX, et al. An armed oncolytic adenovirus system, ZD55-gene, demonstrating potent antitumoral efficacy. Cell Res 2003; 13:481-489.

20 Haridas V, Shrivastava A, Su J, et al. VEGI, a new member of the TNF family activates nuclear factor-kappa B and c-Jun Nterminal kinase and modulates cell growth. Oncogene 1999; 18:6496-6504.

21 Besnault-Mascard L, Leprince C, Auffredou MT, et al. Caspase- 8 sumoylation is associated with nuclear localization.
Oncogene 2005; 24:3268-3273.

22 Stennicke HR, Jurgensmeier JM, Shin H, et al. Pro-caspase-3 is a major physiologic target of caspase-8. J Biol Chem 1998; 273:27084-27090.

23 Yue TL, Ni J, Romanic AM, et al. TL1, a novel tumor necrosis factor-like cytokine, induces apoptosis in endothelial cells. Involvement of activation of stress protein kinases (stressactivated protein kinase and p38 mitogen-activated protein kinase) and caspase-3-like protease. J Biol Chem 1999; 274:1479-1486.

24 Migone TS, Zhang J, Luo X, et al. TL1A is a TNF-like ligand for DR3 and TR6/DcR3 and functions as a T cell costimulator. Immunity 2002; 16:479-492.

25 Liu XY, Qiu SB, Zou WG, et al. Effective gene-virotherapy for complete eradication of tumor mediated by the combination of hTRAIL (TNFSF10) and plasminogen k5. Mol Ther 2005; 11:531-541

26 Yang CR, Hsieh SL, Teng CM, et al. Soluble decoy receptor 3 induces angiogenesis by neutralization of TL1A, a cytokine belonging to tumor necrosis factor superfamily and exhibiting angiostatic action. Cancer Res 2004; 64:1122-1129.

27 Putzer BM, Stiewe T, Parssanedjad K, Rega S, Esche H. E1A is sufficient by itself to induce apoptosis independent of p53 and other adenoviral gene products. Cell Death Differ 2000; 7:177-188.

28 Hanahan D, Weinberg RA. The hallmarks of cancer. Cell 2000; 100:57-70.

29 Zhang Z, Zou W, Wang J, et al. Suppression of tumor growth by oncolytic adenovirus-mediated delivery of an antiangiogenic gene, soluble Flt-1. Mol Ther 2005; 11:553-562.

30 Hermiston TW, Kuhn I. Armed therapeutic viruses: strategies and challenges to arming oncolytic viruses with therapeutic genes. Cancer Gene Ther 2002; 9:1022-1035.

31 Jaffe EA, Nachman RL, Becker CG, Minick CR. Culture of human endothelial cells derived from umbilical veins. Identification by morphologic and immunologic criteria. $J$ Clin Invest $1973 ; \mathbf{5 2}: 2745-2756$.

32 Herrmann M, Lorenz HM, Voll R, et al. A rapid and simple method for the isolation of apoptotic DNA fragments. Nucleic Acids Res 1994; 22:5506-5507.

(Supplementary information is linked to the online version of the paper on the Cell Research website.) 